\title{
Chronic Serous Otitis Media as a Manifestation of Temporal Meningioma
}

\author{
S. Keereweer ${ }^{a}$ R.M. Metselaar ${ }^{a} \quad$ R. Dammers ${ }^{b}$ J.A.U. Hardillo ${ }^{a}$ \\ Departments of a Otorhinolaryngology, Head and Neck Surgery and beurosurgery, Erasmus Medical Center, \\ Rotterdam, The Netherlands
}

\section{Key Words}

Cerebrospinal fluid $\cdot$ Chronic serous otitis media .

Meningioma $\cdot$ Otitis media

\begin{abstract}
Chronic serous otitis media is a common problem in the daily routine of the otorhinolaryngologist. In the majority of cases, the cause is related to dysfunction of the eustachian tube due to viral or bacterial rhinitis and occasionally to nasopharyngeal tumors. We report a case of a patient presenting with chronic serous otitis media that was resistant to conventional therapy. MRI with gadolinium finally revealed that the middle ear fluid was caused by leakage of cerebrospinal fluid (CSF) due to bone destruction by a temporal meningioma. The CSF leakage was closed by surgery. Histopathology confirmed meningioma in the temporal bone.
\end{abstract}

Copyright $\odot 2011$ S. Karger AG, Basel

\section{Introduction}

Chronic serous otitis media is a common problem in the daily practice of the otorhinolaryngologist. The cause is often related to dysfunction of the eustachian tube due to viral or bacterial rhinitis and occasionally to nasopha- ryngeal tumors (e.g. cyst, papilloma, lipoma, polyposis, or carcinoma). We report a case of a patient presenting with a unilateral chronic serous otitis media that was resistant to conventional therapy. MRI with gadolinium finally revealed that the middle ear fluid was caused by leakage of cerebrospinal fluid (CSF) due to bone destruction by a temporal meningioma.

\section{Case Report}

A 45-year-old woman visited our ORL department with hearing loss in her left ear which had begun 2 months earlier. She had no prior history of ear problems. Five years before, she had undergone surgical removal of a meningioma of the right parietal meninges and was currently receiving stereotactic radiotherapy for two other meningiomas: one extending into the right sphenoid sinus and the second on the convexity of the right frontal meninges. No other abnormalities were reported by the radiologist on pre-radiotherapy imaging. Physical examination revealed fluid in the left middle ear without any signs of nasal or nasopharyngeal obstruction. A ventilation tube was placed in the left ear, which resulted in a clear discharge.

During the following months, the patient consistently complained of left ear discharge without fever, despite maximal conservative therapy consisting of local corticosteroids and local and systemic antibiotics. In the meanwhile, she also developed leftsided headaches. For this reason, MRI with gadolinium was performed, which revealed a temporal meningioma on the left side

\section{KARGER}

Fax +4161306 1234 E-Mail karger@karger.ch www.karger.com
(C) 2011 S. Karger AG, Basel

0301-1569/11/0735-0287\$38.00/0

Accessible online at:

www.karger.com/orl
S. Keereweer, MD

Department of Otorhinolaryngology, Head and Neck Surgery

Erasmus Medical Center, PO Box 2040

NL-3000 CA Rotterdam (The Netherlands)

Tel. +31 107040 120, E-Mail s.keereweer@erasmusmc.nl 
Fig. 1. a Axial MRI scan with gadolinium showing the temporal meningioma on the left side (right-pointing arrow). A second meningioma which is being treated with stereotactic radiotherapy is located in the wall of the right sphenoid sinus (leftpointing arrow). b Coronal MRI scan with gadolinium showing the extension of the meningioma in the mastoid bone (arrow).
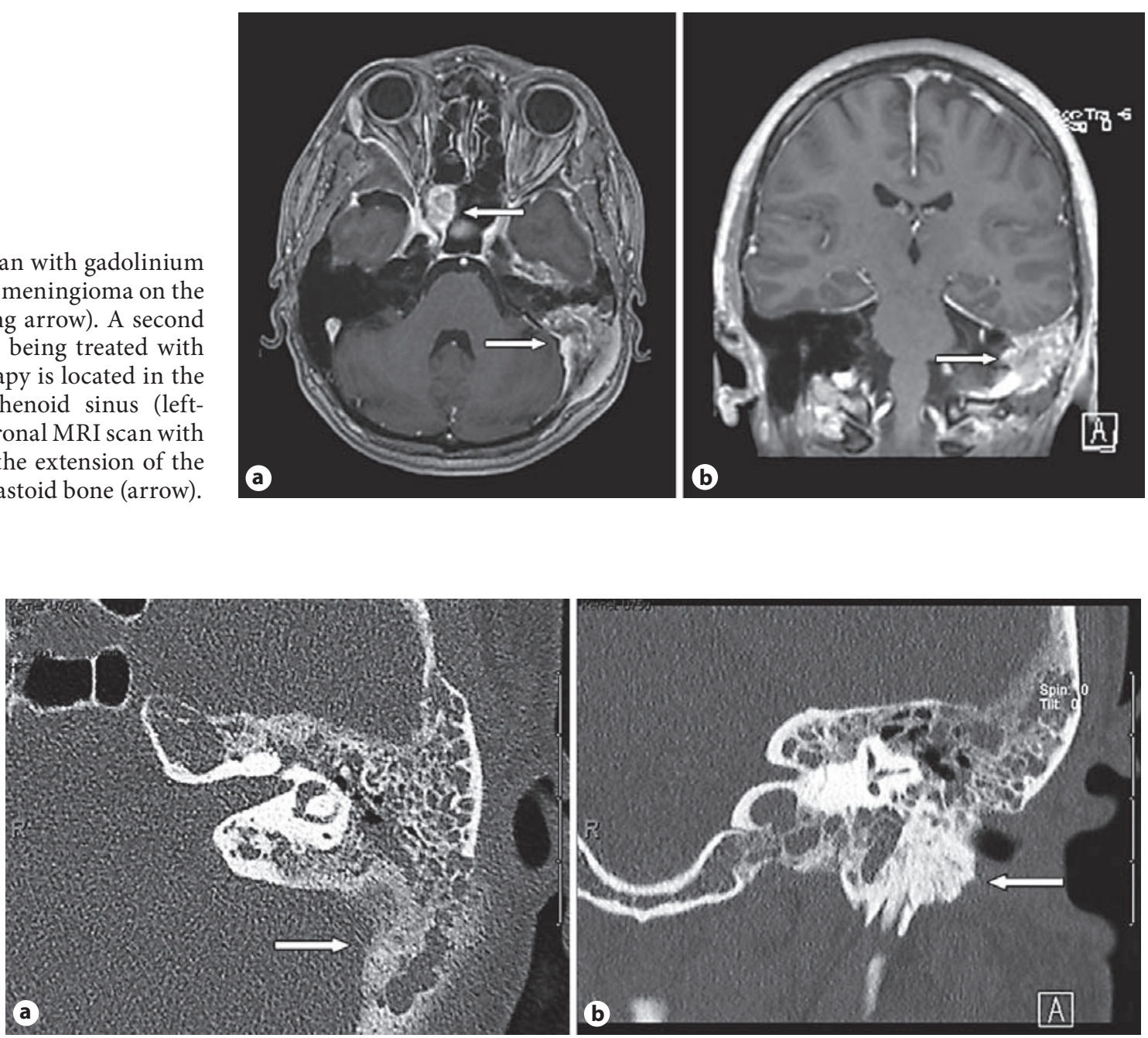

Fig. 2. CT scan illustrating the posterosuperior defect of the left mastoid bone near the sigmoid sinus as a result of destruction by the temporal meningioma. a Axial view. b Coronal view.

that was not detected previously (fig. 1). On a subsequent CT scan of the region, a bone defect was detected in the posterosuperior mastoid bone, which was the suspected route of CSF leakage (fig. 2).

In order to close the CSF leakage, we decided to remove the tumor as much as possible. A subtotal petrosectomy was performed, revealing porous deformation of the mastoid plane (fig. 3a). Hyperplastic mucosa was removed from the middle ear and the bony boundaries of the petrous bone, together with the tympanic tegmen. The cochlea and labyrinth were left intact. The outer part of the temporal bone was removed for histopathology. Subsequently, dura was sealed with TachoSil ${ }^{\circledR}$ and fibrin glue and the cavity was filled with abdominal adipose tissue (fig. 3b). Facial nerve and cochlear functions were preserved. Histopathological analysis of the temporal bone revealed localization of meningioma with bone deformation due to infiltration (fig. 4). One week after surgery, the patient complained of headaches which were caused by postoperative CSF leakage. These complaints resolved after external lumbar CSF drainage over 2 weeks. Postoperatively, the patient received stereotactic radiotherapy on the remaining left temporal meningioma with $54 \mathrm{~Gy}$ in 30 fractions.

\section{Discussion}

Chronic serous otitis media is frequently encountered in the daily routine of an otorhinolaryngologist. However, it is rarely the initial presentation of a temporal meningioma. Meningiomas arise from the dural coverings of the brain. They are slow-growing benign tumors which account for $13-26 \%$ of all primary intracranial tumors 

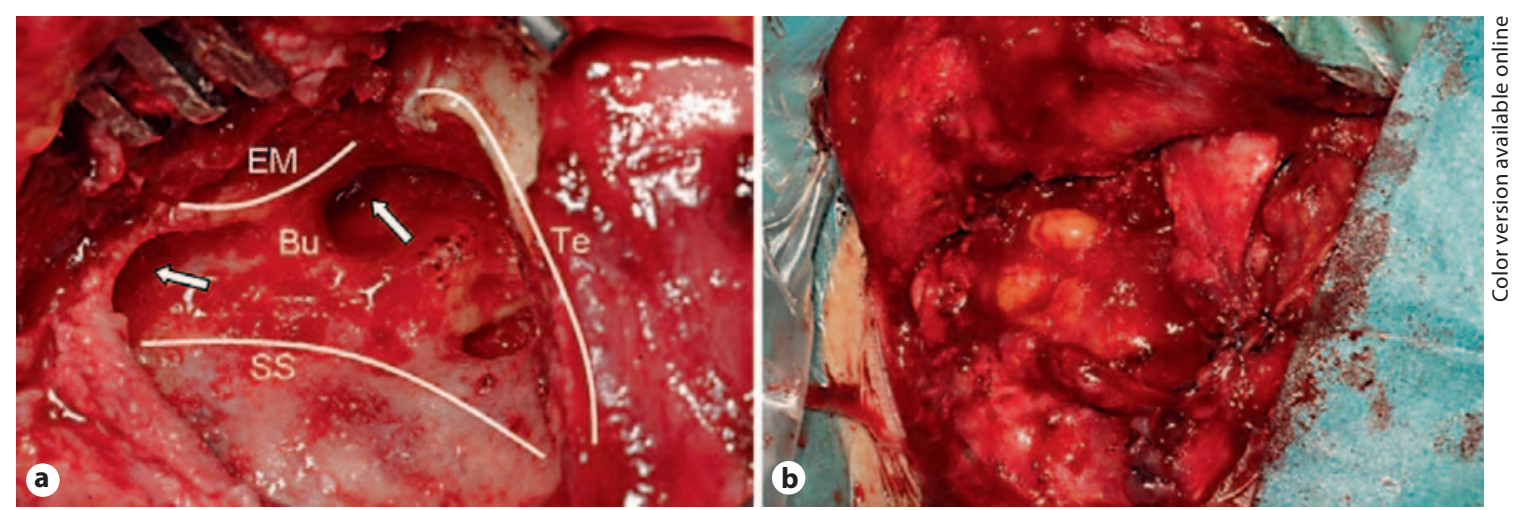

Fig. 3. Surgical closure of CSF leakage (left ear). a Situation after complete mastoidectomy, atticoantrotomy, and exposure of the tegmen tympani. The external meatus (EM), buttress ( $\mathrm{Bu})$, tegmen (Te), and sigmoid sinus (SS) are shown. Arrows indicate the tip of the mastoid (left arrow) and the antrum (right arrow). b The cavity was closed with abdominal adipose tissue.

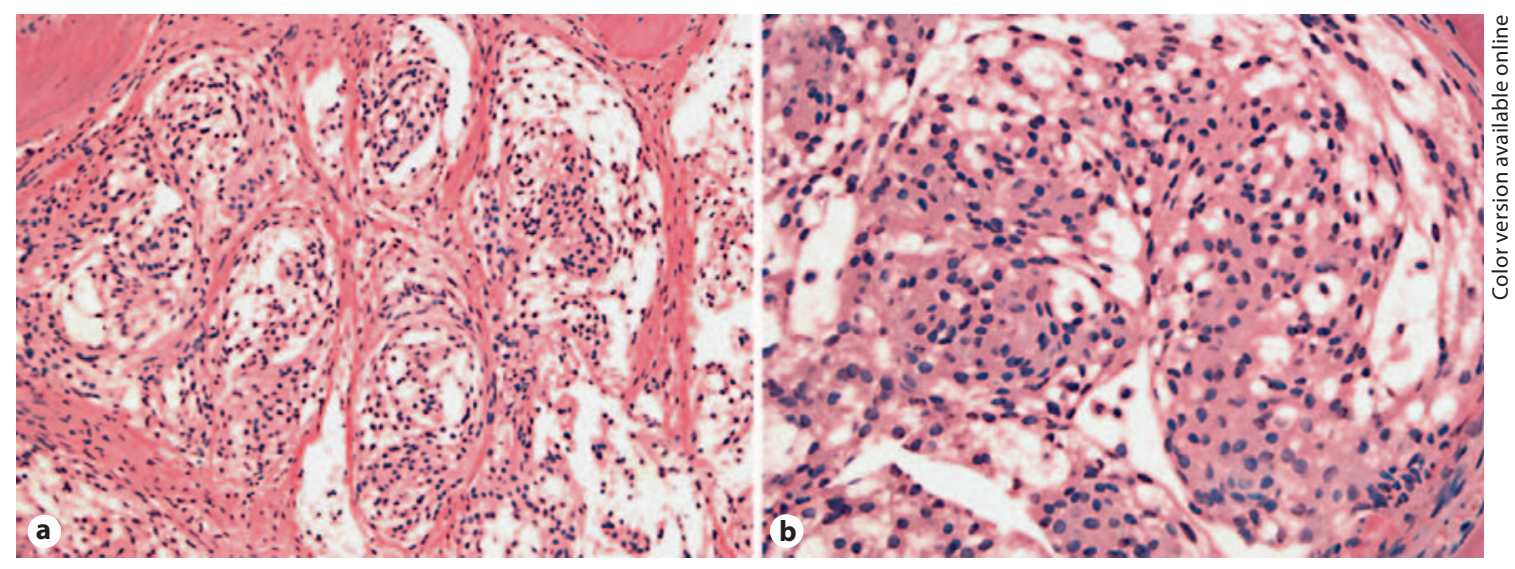

Fig. 4. Histopathology of temporal bone with localization of meningioma. Polygonal epithelioid cells including eosinophilic cytoplasm and small oval nuclei containing a widely spread chromatin pattern are shown. $\mathbf{a} \times 10$. b $\times 20$.

[1]. In most cases, otologic and neurotologic symptoms of temporal meningioma are dizziness, vertigo, sensorineural hearing loss, tinnitus, or facial palsy caused by the compression of the VII or VIII cranial nerves.

In very few cases, extension of meningiomas into the mastoid or middle ear cleft has been described. In these patients, unilateral chronic otitis media had also been the initial presentation [2-4]. Ayache et al. [5] described a series of patients in whom the delay between the onset of symptoms suggesting serous otitis media and diagnosis of temporal meningioma ranged between 1 and 10 years. Before the diagnosis of meningioma was made, most of their patients underwent various medical treatments or surgical procedures for unilateral serous otitis media. In particular, a common sign was severe episodes of discharge after ventilation tube insertion.

Leakage of CSF into the middle ear or extension of meningiomas in the temporal bone can occur via defects of the tegmen tympani, posterior fossa plate, internal auditory canal, or jugular foramen [6]. In the current case, a CT scan revealed the leakage pathway through a posterosuperior defect of the mastoid bone near the sigmoid sinus as a result of destruction by the temporal meningioma (fig. 2). In our patient, the initial diagnosis was made by MRI with gadolinium as a contrast agent, which is the imaging modality of choice to confidently deter- 
mine the diagnosis and extent of a temporal bone meningioma [1]. This provides a potential dilemma because first-line imaging of chronic serous otitis media is performed by CT without contrast injection [7], by which detection of intracranial meningioma is much more difficult. Nevertheless, in accordance with others [5], we feel that complementary imaging is indicated in patients with chronic unilateral serous otitis media not responding to conservative therapy or ventilation tube insertion, and in whom no signs of a nasopharyngeal tumor are found by fiberscopy.

\section{Conclusion}

Leakage of CSF into the middle ear can be the initial presentation of temporal meningioma. These patients can present with therapy-resistant unilateral chronic se- rous otitis media. Considering the impact on the patient of the diagnosis, combined with a delay of 1-10 years between onset of ear complaints and disclosure of temporal meningioma described in the literature [5], complementary imaging is indicated in patients with chronic serous otitis media without nasopharyngeal obstruction.

\section{Acknowledgement}

We thank Dr. R.D. Van Baare-Georgieva (Department of Pathology, Erasmus Medical Center) for providing us with the histological analysis.

\section{Disclosure Statement}

The authors declare that they have no conflict of interest.

\section{References}

1 Whittle IR, Smith C, Navoo P, et al: Meningiomas. Lancet 2004;363:1535-1543.

-2 Marcelissen TA, de Bondt RB, Lammens M, et al: Primary temporal bone secretory meningioma presenting as chronic otitis media. Eur Arch Otorhinolaryngol 2008;265:843846.
>3 Cenacchi G, Ferri GG, Salfi N, et al: Secretory meningioma of the middle ear: a light microscopic, immunohistochemical and ultrastructural study of one case. Neuropathology 2008;28:69-73.

4 George M, Ikonomidis C, Pusztaszeri M, et al: Primary meningioma of the middle ear: case report. J Laryngol Otol 2010;124:572574.

5 Ayache D, Trabalzini F, Bordure P, et al: Serous otitis media revealing temporal en plaque meningioma. Otol Neurotol 2006;27: 992-998.
6 Chang CY, Cheung SW, Jackler RK: Meningiomas presenting in the temporal bone: the pathways of spread from an intracranial site of origin. Otolaryngol Head Neck Surg. 1998;119:658-664.

$\checkmark 7$ Williams MT, Ayache D: Imaging of the postoperative middle ear. Eur Radiol 2004; 14:482-495. 